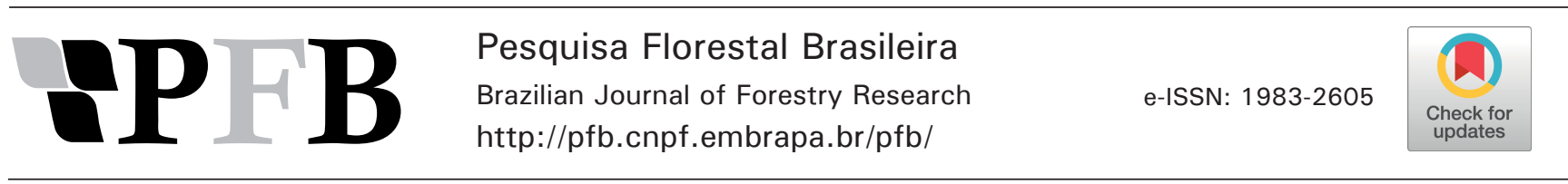

\title{
O licenciamento de Planos de Manejo Florestal da Caatinga assegura sua sustentabilidade?
}

\author{
Isabelle Maria Jacqueline Meunier ${ }^{1 *(D)}$, Rinaldo Luiz Caraciolo Ferreira ${ }^{1 D}$, José Antônio Aleixo da Silva ${ }^{1}$ (D) \\ ${ }^{1}$ Universidade Federal Rural de Pernambuco, Av. Dom Manuel de Medeiros, s/n, CEP 52171-900, Dois Irmãos, Recife, PE
}

"Autor correspondente:

isabelle.meunier@ufrpe.br

Termos para indexação:

Gestão florestal

Recursos naturais

Produção florestal

Index terms:

Forest management

Natural resources

Forestry production

Histórico do artigo:

Recebido em 31/05/2017

Aprovado em 12/07/2018

Publicado em 19/10/2018

doi: 10.4336/2018.pfb.38e201701461

\begin{abstract}
Resumo - Objetivou-se identificar as dimensões de sustentabilidade passíveis de serem avaliadas em planos de manejo florestal (PMF) da Caatinga e descrever quais delas são melhor contempladas em PMF licenciados e ativos. Para isso, tomou-se uma amostra de 40 PMF licenciados pelo órgão ambiental de Pernambuco, analisando-se o roteiro recomendado pelo órgão e o atendimento aos itens propostos. A dimensão técnica, com descrição de aspectos quantitativos do material lenhoso, é a melhor descrita, embora tenha obtido apenas $23 \%$ de atendimento satisfatório. Aspectos sociais não são contemplados nos estudos apresentados e critérios das dimensões ambiental e econômica são fracamente observados. As exigências normativas para elaboração de PMF na Caatinga não oferecem elementos para avaliação da sustentabilidade da atividade e os PMF mostram baixo nível de atendimento às exigências.
\end{abstract}

\section{The environmental licensing of forest management plans in Caatinga ensures their sustainability?}

\begin{abstract}
We intend to identify the sustainability dimensions that can be evaluated in forest management plans (FMP) of natural vegetation of Caatinga and to describe which of those are best considered in licensed and active plans. The guidelines recommended by the environmental agency was analyzed in a sample of 40 FMP licensed in Pernambuco, Brazil, observing the attendance to the proposed items. The technical dimension, with description of quantitative aspects of the timber production is the best described, although it has only achieved $23 \%$ according to the requirements. Social aspects are not contemplated in the plans and criteria of the environmental and economic dimensions are poorly observed. The normative requirements for the elaboration of a FMP of natural vegetation of Caatinga do not offer elements to evaluate the sustainability and the plans show low level of attendance to the requirements.
\end{abstract}

\section{Introdução}

A exploração extrativista da vegetação natural da Caatinga objetiva atender a demanda por dendrocombustíveis (lenha e carvão) e o manejo florestal é apresentado como alternativa legal e conservacionista para essa exploração, acrescentando-lhe epíteto de "sustentável" em função da observância de exigências normativas.
No Brasil, o manejo florestal sustentável encontra previsão na Lei $\mathrm{n}^{\circ} 12.651$ de 25 de maio de 2012, devendo ser realizado a partir de fundamentos técnicos e científicos (Brasil, 2012). Especificações desses fundamentos estão presentes em normas estaduais e são diferentes nos biomas Cerrado, Amazônia e Caatinga.

A sustentabilidade da produção florestal era concebida, até meados do Séc. XX, como a simples produção madeireira sustentável. No entanto, como 
destacam Karvonen et al. (2017), compreende-se a sustentabilidade como uma combinação de aspectos ambientais, econômicos e sociais, exigindo avaliações de impactos multidimensionais.

As condições básicas para a sustentabilidade do manejo florestal passam pelo planejamento adequado, baseado em peculiaridades de cada local, e nas avaliações que permitam estabelecer o ciclo de corte e as influências de práticas culturais durante este ciclo, além de considerar aspectos econômicos e sociais (Schneider \& Finger, 2000). Todos esses aspectos devem ser cuidadosamente investigados ao manejar florestas produtivas, definidas por Zarin (2005) como aquelas utilizadas economicamente, objetivando promover a sustentabilidade ecológica, econômica e social, sem apresentar problemas quanto à regeneração natural.

As dimensões técnica, ambiental, econômica e social da sustentabilidade da atividade devem estar descritas, de alguma forma, em um plano de manejo florestal (PMF), documento técnico considerado como um tipo de estudo ambiental (Conselho Nacional do Meio Ambiente, 1997), que traz os diagnósticos realizados e as decisões técnicas necessárias à condução e à avaliação da prática do manejo, para que se possa avaliar o atendimento de critérios básicos para a sustentabilidade. Por sua vez, o licenciamento do PMF exige a descrição de aspectos que podem estar ou não relacionados a esses critérios de sustentabilidade.

Assim, o fato da exploração florestal se dar por meio de PMF licenciados não parece ser condição suficiente para garantir algum grau de sustentabilidade à atividade. O objetivo deste trabalho foi analisar os itens solicitados no processo de licenciamento de PMF na Caatinga, avaliando se esses constituem critérios que permitam avaliações de sustentabilidade e estimar, em uma amostra de PMF, o nível de atendimento a esses critérios.

\section{Material e métodos}

Para identificar as dimensões da sustentabilidade que podem ser avaliadas na análise técnica dos planos de manejo florestal (PMF), relacionaram-se os itens integrantes do roteiro recomendado na Instrução Normativa $n^{\circ} 7 / 2006$ da Agência Estadual de Meio Ambiente de Pernambuco (CPRH) (Pernambuco, 2006), que solicita informar: objetivos e metas do plano de manejo; justificativa; uso atual do solo e caracterização do meio; aspectos técnicos, princípios e critérios (entre eles, inventário florestal e cubagem, estoque atual, regeneração, restrições ao corte, ciclo e modalidade de corte, técnicas de exploração, tamanho dos talhões e infraestrutura); monitoramento; impactos ambientais e medidas mitigadoras; viabilidade econômica, finalizando com a inclusão de memória de cálculo e análise estatística (Pernambuco, 2006).

Procurou-se associar os itens requisitados às dimensões técnica, ecológica, econômica e social da sustentabilidade. Foram propostos critérios de avaliação para cada item do roteiro, avaliando-os em uma amostra de 40 PMF licenciados pela CPRH entre 2007 e 2011, selecionados em função da disponibilidade de acesso à documentação, entre 68 PMF ativos no período.

$\mathrm{O}$ atendimento a cada um dos critérios foi julgado como aceitável (nota 2), regular (parcialmente atendido, nota 1) ou não aceitável ou não apresentado (nota 0), conforme adaptação de referencial de respostas adotado pela FAO na identificação de casos exemplares de manejo na América Latina e Caribe (FAO, 2010). Foram calculadas as notas médias de cada critério, o índice de atendimento ao critério (equação 1) e o índice médio de atendimento à dimensão (equação 2).

$$
\begin{aligned}
& \mathrm{IA}_{\mathrm{C}}(\%)=\frac{\text { Nota } * 100}{2} \\
& \mathrm{IA}_{\mathrm{D}}(\%)=\overline{\mathrm{IA}}_{\mathrm{C}_{\mathrm{I}}}
\end{aligned}
$$

Em que: $\mathrm{IA}_{\mathrm{c}}=$ índice de atendimento ao critério; $\mathrm{IA}_{\mathrm{D}}=$ índice médio de atendimento à dimensão; $\mathrm{c}_{\mathrm{i}}$ é o i-ésimo critério da dimensão D.

Os 40 PMS estiveram entre os analisados por Meunier (2014), localizados na região semiárida pernambucana, com áreas manejadas entre 31,75 ha a $1.100,00$ ha, e talhões de corte anual entre de 3,1 ha a 73,3 ha, sendo 8 do tipo plano de manejo florestal simplificado simultâneo, com um único talhão inferior a 50 ha, e 13 podem ser considerados comunitários, desenvolvidos por associações de produtores ou assentados da reforma agrária.

\section{Resultados}

A dimensão técnica dos planos de manejo florestal (PMF) foi o item mais representado, entre os exigidos pelas normas do órgão ambiental responsável, presente na descrição de objetivos e metas (Tabela 1). No 
detalhamento deveria constar a apresentação do uso do solo atual e projetado, aspectos técnicos, princípios e critérios, incluindo aspectos metodológicos do inventário, resultados obtidos, definições de ciclo e modalidade de corte e técnicas de exploração e de condução da regeneração da vegetação (Tabela 1). Além desses, os métodos e técnicas propostos para monitoramento deveriam ser explicados, embora não seja necessário especificar o objeto do monitoramento.

Tabela 1. Dimensões de sustentabilidade presentes no roteiro para elaboração de planos de manejo florestal em Pernambuco.

Table 1. Sustainability dimensions present in the guidelins for forest management plans elaboration in Pernambuco.

\begin{tabular}{cl}
\hline Dimensão & \multicolumn{1}{c}{ Itens do roteiro* $^{*}$} \\
& Objetivos e metas \\
& Planejamento do uso do solo \\
Técnica & Metodologia do inventário \\
& Informações sobre estoque e crescimento \\
& Técnicas e práticas do manejo \\
& Métodos e técnicas de monitoramento \\
\hline & Caracterização dos meios físico e biológico \\
& Relação de espécies arbóreas \\
& Restrições de corte definidas em função de espécies \\
Ecológica & raras e protegidas, limitações edáficas, etc. \\
& Descrição dos impactos ambientais \\
& Descrições das medidas mitigadoras \\
& Métodos e técnicas de monitoramento \\
\hline Econômica & Viabilidade econômica \\
\hline Social & Não há \\
\hline *Conforme Instrução Normativa CPRH nº/2006 (Pernambuco, 2006).
\end{tabular}

Não são especificados indicadores de viabilidade econômica, sendo apenas solicitado que os custos das operações e preços dos produtos sejam considerados. A dimensão social não é considerada, a menos que os elaboradores a incluam na justificativa do projeto.

Os 20 critérios elaborados, associados às três dimensões de sustentabilidade, encontram-se na Tabela 2, com os respectivos índices de atendimento na amostra avaliada.

Em relação à dimensão técnica ( $23 \%$ de atendimento), os objetivos apresentados foram genéricos (manejar a Caatinga) e inapropriados (preservar a Caatinga) e restringiram-se à produção madeireira destinada à lenha e carvão. O objeto da produção foi lenha (25 PMF) e carvão (14 PMF). Dois projetos previram a produção simultânea de lenha e carvão, sem considerar que um se tratava de transformação do outro. Apenas um PMF amostrado buscou quantificar, além do carvão a ser produzido, outros produtos madeireiros (estacas e mourões) e não madeireiros (cascas).

As metas, quando descritas, foram insuficientemente definidas, sem especificação de expectativa de produção ou renda nem definição de mercado. Por outro lado, os PMF analisados apresentaram informações satisfatórias referentes ao planejamento do uso de terras da propriedade, em função da legislação vigente e usos atuais. No entanto, não foram fornecidas informações adequadas à avaliação da adequação do uso proposto às potencialidades e restrições ambientais da área.

A descrição da metodologia do inventário florestal permitiu conhecer os métodos e técnicas empregados, predominando o processo aleatório de seleção, com unidades amostrais com $400 \mathrm{~m}^{2}$. A intensidade amostral adotada variou de $0,04 \%$ a $1,15 \%$ e, embora o esforço amostral tenha sido em muitos casos muito baixo ( 5 ou 6 parcelas), em todos os PMF foram informados erros de amostragem inferiores a $20 \%$.

Foram identificadas várias inconsistências nos estoques estimados e, em alguns casos, informações de área basal e densidade não foram apresentadas. $O$ fator de forma 0,9 para estimar o volume real foi utilizado por $80,5 \%$ dos PMF analisados, adotando-se nos demais equações volumétricas desenvolvidas pelo Projeto PNUD/FAO (Zakia et al., 1990). O fator de empilhamento de $3,32 \mathrm{st} \mathrm{m}^{-3}$ foi adotado em todos os PMF.

Não se apresentaram estimativas locais de crescimento obtidas de fontes atualizadas e em condições semelhantes, nem mesmo de outros sítios. A definição do ciclo de corte obedeceu ao mínimo estabelecido pela norma estadual (15 anos) e não se consideraram outros usos potenciais das espécies lenhosas. Apenas um PMF cogitou a exploração da casca do angico para extração do tanino, sem esclarecer as condições de extração e comercialização.

O corte raso foi o tipo de corte adotado por todos os PMF, alguns deles apontando restrições de espécies protegidas ou definidas em função de deliberações técnicas não especificadas. A menor intensidade de corte adotada foi $64 \%$ do volume total, sendo que apenas cinco PMF apresentaram intensidade de corte inferior a $80 \%$ do volume estimado. 
Tabela 2. Atendimento a critérios de sustentabilidade em planos de manejo licenciados em Pernambuco, em 2014.

Table 2. Compliance with sustainability criteria in management plans licensed in Pernambuco, in 2014.

\begin{tabular}{|c|c|c|c|c|}
\hline Dimensão & Critério & 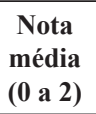 & $\begin{array}{l}\mathbf{I} \mathbf{A}_{\mathrm{C}} \\
\mathbf{\%}\end{array}$ & $\begin{array}{l}\mathbf{I A}_{\mathrm{D}} \\
\%\end{array}$ \\
\hline \multirow{11}{*}{ 苞 } & $\begin{array}{l}\text { Os objetivos são claros, precisos e suficientemente flexíveis para se adequarem a } \\
\text { mudanças ao longo do desenvolvimento do plano e permitem observar o potencial } \\
\text { de produtos e serviços e as alternativas de múltiplos usos. }\end{array}$ & 0,7 & 33 & \multirow{11}{*}{23} \\
\hline & $\begin{array}{l}\text { As metas são objetivas e quantificadas em função dos diagnósticos e das } \\
\text { oportunidades. }\end{array}$ & 0,4 & 19 & \\
\hline & Considera a legislação e os demais usos da propriedade. & 1,6 & 81 & \\
\hline & Leva em conta as potencialidades e restrições ambientais. & 0,0 & 0 & \\
\hline & $\begin{array}{l}\text { Descreve métodos e técnicas adequados à obtenção, análise e apresentação de } \\
\text { dados para análise técnica. }\end{array}$ & 1,5 & 75 & \\
\hline & Apresenta estimativas de estoque precisas e fidedignas. & 0,8 & 42 & \\
\hline & $\begin{array}{l}\text { Apresenta estimativas de crescimento que permitam estabelecer a possibilidade de } \\
\text { aproveitamento anual. }\end{array}$ & 0,0 & 0 & \\
\hline & Definição do ciclo de corte em função do incremento e dos objetivos. & 0,0 & 0 & \\
\hline & Colheita de espécies determinada pelos seus usos potenciais. & 0,0 & 0 & \\
\hline & $\begin{array}{l}\text { Tipo e intensidade de corte definidos em função das características da área e dos } \\
\text { resultados do inventário. }\end{array}$ & 0,1 & 3 & \\
\hline & $\begin{array}{l}\text { Constam variáveis, periodicidade e métodos de monitoramento de crescimento de } \\
\text { área em regeneração e da vegetação a explorar. }\end{array}$ & 0,0 & 0 & \\
\hline \multirow{6}{*}{ 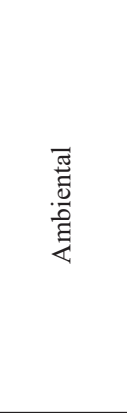 } & $\begin{array}{l}\text { Diagnóstico com informações sobre tipos e aptidão do solo, processos erosivos, } \\
\text { corpos d'água temporários e permanentes, fauna e flora, espécies indicadoras de } \\
\text { qualidade ambiental, de valor científico e econômico, raras e ameaçadas, áreas de } \\
\text { preservação permanente e outros sítios de especial valor ecológico. }\end{array}$ & 0,2 & 8 & \multirow{6}{*}{7} \\
\hline & Identificação das espécies lenhosas e caracterização quanto aos usos potenciais. & 0,2 & 8 & \\
\hline & Definição das restrições de corte em função dos objetivos e do diagnóstico. & 0,6 & 28 & \\
\hline & $\begin{array}{l}\text { Identificação, previsão de magnitude e interpretação dos prováveis impactos nos } \\
\text { meios físico, biológico e socioeconômico. }\end{array}$ & 0,0 & 0 & \\
\hline & Definição das medidas mitigadoras para os impactos identificados. & 0,0 & 0 & \\
\hline & Programa de acompanhamento e monitoramento dos impactos. & 0,0 & 0 & \\
\hline \multirow{3}{*}{ 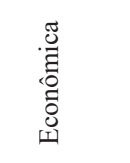 } & $\begin{array}{l}\text { Identificação dos componentes de custos (incluindo encargos e remuneração da } \\
\text { mão-de-obra) e análise de preços dos produtos no mercado. }\end{array}$ & 0,6 & 31 & \multirow{3}{*}{12} \\
\hline & Estimativas do ponto de equilíbrio de produção e de rentabilidade. & 0,1 & 6 & \\
\hline & Mecanismos de registro e controle de despesas, produção e receitas. & 0,0 & 0 & \\
\hline
\end{tabular}

$\mathrm{IA}_{\mathrm{c}}$ : índice de atendimento ao critério; $\mathrm{IA}_{\mathrm{d}}$ : índice de atendimento à dimensão.

Em todos os PMF foi esperada a recomposição da vegetação por meio da rebrota de cepas e por sementes, embora não se apresentem informações a respeito da eficácia do método. Não se descreveram métodos e técnicas de monitoramento de regeneração e crescimento.

$\mathrm{O}$ atendimento aos critérios da dimensão ambiental foi, em geral, insatisfatório, obtendo o menor índice de atendimento (7\%). Embora o item referente aos diagnósticos do meio físico e biológico tenha estado sempre presente nos documentos, a análise do conteúdo mostrou que em $80 \%$ dos PMF eram baseados em dados secundários e sem referências. Não foram encontradas descrições de métodos de obtenção de informações sobre fauna, que parecem ter sido resultado de depoimentos de moradores locais, sem registros de dados primários. A fauna citada foi, na maioria das vezes, repetida de forma similar em vários PMF. Na descrição da flora arbórea, foi possível observar diversas imprecisões na classificação e equívocos na nomenclatura botânica das espécies.

Todos os PMF analisados apresentaram itens intitulados "impactos ambientais e medidas mitigadoras", não sendo adotados, no entanto, procedimentos para identificação e interpretação de impactos ambientais 
nem descrição de medidas mitigadoras e programa de acompanhamento e monitoramento de impactos, como esperado em estudos ambientais. Quando citadas, as medidas mitigadoras restringiam-se ao não uso de motosserra e do fogo.

Para informar sobre a viabilidade econômica, foram apresentadas estimativas de custos e previsão de preços dos produtos por $56 \%$ dos PMF avaliados. A maioria não realizou análises econômicas simples e desconsiderou, por exemplo, custos de construção, enchimento e esvaziamento dos fornos para carvão. Nenhum dos PMF considerou o custo da terra nas análises econômicas e não foram incluídos impostos, encargos sociais ou taxas. A remuneração da mão-de-obra, quando considerada, resumiu-se às operações de corte e baldeio da lenha.

Não houve análise de rentabilidade ou estimativas de ponto de equilíbrio entre custos e receitas e não foram informados instrumentos de registro e análise de despesas, produção e receitas.

\section{Discussão}

Ter a lenha como único objetivo dos planos de manejo florestal (PMF) da Caatinga reflete uma tendência da produção florestal imperante até o século $\mathrm{XX}$, cujo foco exclusivo na produção madeireira em regime de rendimentos sustentados determinava a simplificação e homogeneização da estrutura e das operações florestais (Messier \& Puettmann, 2011).

Seguindo essa tendência, os PMF de Caatinga são voltados à quantificação do componente lenhoso para a obtenção de autorização de exploração (AutEx), não se encontrando informações sobre a ocorrência e distribuição das fitofisionomias, estado de conservação e interações da vegetação com a fauna ou com os componentes do meio físico. A desconsideração dos impactos ambientais e das medidas mitigadoras nos planos de manejo da vegetação natural foi também observada por Coelho et al. (2008), na análise documental de planos de manejo de Minas Gerais. O desconhecimento dos impactos da exploração florestal por meio do corte raso nos meios físicos e biológicos na região semiárida é agravado pela inexistência de dados de monitoramento.

Os PMF em andamento, em Pernambuco, não adotam estimativas de crescimento que levem em conta os fatores do sítio, assumindo indistintamente o ciclo de corte de 15 anos. Burger (1980) alerta que qualquer generalização no manejo de florestas, inclusive no ciclo de corte, pode produzir efeitos danosos a um povoamento florestal. Braz et al. (2005) destacam que há frequente confusão entre ciclo mínimo (salvaguarda da legislação) e ciclo ideal calculado, enfatizando que o ciclo não deveria ser o mesmo para todos os planos de manejo.

Os valores de incremento médio anual (IMA) que proporcionariam o reestabelecimento do estoque lenhoso após 15 anos foram superiores àqueles obtidos em diferentes localidades da Caatinga, sistematizados por Ferreira et al. (2010), que variaram de $0,45 \mathrm{~m}^{3} \mathrm{ha}^{-1} \mathrm{ano}^{-1}$ a $1,34 \mathrm{~m}^{3} \mathrm{ha}^{-1} \mathrm{ano}^{-1}$, em relação ao volume cilíndrico, equivalentes a valores inferiores a 4,5 st.ha ${ }^{-1}$ ano $^{-1}$. Ainda assim, se encontram na ampla faixa apresentada por Riegelhaupt et al. (2010), também referentes a volume cilíndrico (entre $0,3 \mathrm{~m}^{3} \mathrm{ha}^{-1} \mathrm{ano}^{-1} \mathrm{e}$ $10,9 \mathrm{~m}^{3} \mathrm{ha}^{-1} \mathrm{ano}^{-1}$ ). Note-se que, em ambos os casos, não se apresentam resultados de Pernambuco. Conforme os resultados de Riegelhaupt et al. (2010), em apenas dois locais, no Ceará, houve registros de IMA superiores a $5,0 \mathrm{~m}^{3} \mathrm{ha}^{-1} \mathrm{ano}^{-1}$. Portanto, é improvável que valores tão altos de IMA ocorram em Pernambuco, permitindo supor que não haverá recuperação do estoque após o ciclo de 15 anos, como alertam Ferraz et al. (2014).

As técnicas de exploração adotadas visam atender ao mercado de lenha e são definidas de forma a obter o máximo aproveitamento do material lenhoso, sem considerar outros usos potenciais das espécies, a despeito das diferenças de potencial energético do material lenhoso (Meunier et al., 2015).

Não se adotam tratamentos silviculturais para promoção da regeneração nem métodos de monitoramento da regeneração e crescimento. Traçando o cenário dos PMF desenvolvidos na Amazônia, Braz (2010) observou a desconsideração dos tratamentos silviculturais que poderiam elevar o incremento periódico anual e afirmou que a taxa anual de extração raramente é fundamentada em dados sobre a estrutura da floresta e no ritmo de crescimento das espécies, ressaltando ainda que as normas exigidas para o manejo das florestas limitam-se a formalidades burocráticas e são pouco exigentes nas questões que garantam a real recuperação das florestas naturais tropicais, o que pode ser considerado válido para a exploração da vegetação natural da Caatinga.

A adoção do corte raso agrava a preocupação com a manutenção da capacidade de recuperação da vegetação. A prática do manejo da vegetação natural fundamentase nas propriedades de resistência e resiliência da 
comunidade, ou seja, na forma como a comunidade reage à perturbação (colheita) e nos processos que ocorrem para a sua recuperação. Uma perturbação que produza um grande impacto pode transformar severamente uma comunidade a ponto de incapacitá-la de retornar a seu estado original, sendo então substituída por uma comunidade diferente (Smith \& Smith, 2001).

A manutenção contínua da cobertura florestal é uma tendência do manejo florestal mundial, em função da maior oferta de serviços ecossistêmicos que o sistema pode fornecer. Pukkala (2016) comprovou isso em florestas da Finlândia e destacou que a conclusão pode ser generalizada para outras regiões, desde que ali se identifiquem os serviços ecossistêmicos mais importantes.

A inexistência de estimativas de indicadores econômicos no PMF como valor presente líquido, valor esperado da terra ou taxa interna de retorno impede a comparação de alternativas de manejo e a verificação da viabilidade da atividade, como feito por Oliveira et al. (2002), no Cerrado, e Timofeicyzyk Junior et al. (2007, 2008), na Amazônia. A renda média anual pode ser estimada como por Francelino et al. (2003), que encontraram valores muito baixos de receitas líquidas por família em assentamento com PMF no Rio Grande do Norte. Apesar de constatar a baixa rentabilidade e o caráter complementar do manejo como atividade produtiva, os autores destacaram a importância da atividade por se constituir em opção de geração de renda em assentamentos sem infraestrutura produtiva.

Marques et al.(2011), também emárea de assentamento, defenderam a viabilidade do manejo florestal, embora tenham observado que o trabalho fora do assentamento e os programas sociais do governo tiveram maior importância do que as atividades produtivas locais. O manejo florestal em assentamentos de Pernambuco representa um diferencial na sua dinâmica financeira, mas os resultados indicam que isso se dá devido baixa remuneração da agricultura e da pecuária, já que à receita anual da atividade é muito baixa (Gomes et al., 2013). Marques et al. (2011) e Gomes et al. (2013) não adotaram indicadores de viabilidade econômica, mas consideraram a relação entre receitas e custos, não incluindo o custo de oportunidade da terra ou os custos de elaboração de projetos e assistência técnica.

A dimensão social, relativa à geração de empregos, condições de trabalho e repartição dos benefícios, não são solicitadas pelo órgão ambiental nem informadas nos PMF, comprometendo a análise, já que esse aspecto é fundamental para a compreensão dos impactos locais à qualidade de vida. Apesar da relevância, a dimensão social é a menos estudada das dimensões da sustentabilidade (Karkoven et al., 2017).

\section{Conclusões}

As exigências normativas para elaboração de planos de manejo florestal (PMF) de Caatinga não oferecem elementos para avaliação integral da sustentabilidade da atividade, enfatizando aspectos técnicos e desconsiderando a dimensão social.

Os PMF em execução mostram baixo nível de atendimento às exigências normativas, notadamente no que diz respeito aos critérios referentes às dimensões ambientais e econômicas. Aspectos sociais não são solicitados nem informados.

O licenciamento de planos de manejo da vegetação nativa da Caatinga isenta a obrigatoriedade de reposição florestal, mas não se constitui em instrumento que garanta a sustentabilidade da atividade e contribua para a gestão do recurso florestal com foco nas suas funções sociais, econômicas e ambientais.

\section{Referências}

Brasil. Lei no 12.651 de 25 de maio de 2010. Dispõe sobre a proteção da vegetação nativa; altera as Leis n ${ }^{\circ} 6.938$, de 31 de agosto de 1981 , 9.393, de 19 de dezembro de 1996, e 11.428, de 22 de dezembro de 2006; revoga as Leis $n^{\circ} 4.771$, de 15 de setembro de 1965, e 7.754, de 14 de abril de 1989, e a Medida Provisória n 2.166-67, de 24 de agosto de 2001; e dá outras providências. Diário Oficial [da] República Federativa do Brasil, 25 de maio de 2012. Disponível em: <http://www.planalto.gov.br/ccivil_03/_Ato2011-2014/2012/ Lei/L12651.htm>. Acesso em: 15 nov. 2016.

Braz, E. M. et al. Critérios para o estabelecimento de limites da intensidade de corte em florestas naturais tropicais. Colombo: Embrapa Florestas, 2005. (Embrapa Florestas. Circular técnica, 101).

Braz, E. M. Subsídios para o planejamento do manejo de florestas tropicais da Amazônia. 2010. 235 f. Tese (Doutorado em Manejo Florestal) - Universidade Federal de Santa Maria, RS.

Burger, D. Ordenamento florestal I: a produção florestal. Curitiba: FUPEF, 1980.

Coelho, D. J. S. et al. Análise documental e de consistência técnica de planos de manejo florestal em áreas de formações florestais no estado de Minas Gerais. Revista Árvore, v. 32, n. 1, p. 69-80, 2008.

Conselho Nacional do Meio Ambiente (Brasil). Resolução no 001 , de 23 de janeiro de 1986. Disponível em: <http://www.mma.gov. br/port/conama/res/res86/res0186.html $\geq$. Acesso em: 30 maio 2017. 
Conselho Nacional do Meio Ambiente (Brasil). Resolução n 237, de 19 de dezembro de 1997. Dispõe sobre a revisão e complementação dos procedimentos e critérios utilizados para o licenciamento ambiental. Disponível em: <http://www.mma.gov.br/port/conama/ legislacao/CONAMA_RES_CONS_1997_237.pdf>. Acesso em: 30 maio 2017.

FAO. Casos ejemplares para el fortalecimento de politicas y programas de manejo florestal sostenible em America Latina y Caribe. Rome, 2010. Disponível em: <http://www.fao.org/fileadmin/ user_upload/training_material/docs/casejes.pdf $>$. Acesso em: 30 maio 2017.

Ferraz, J. S. F. et al. Estrutura do componente arbustivo-arbóreo da vegetação em duas áreas de caatinga, no município de Floresta, Pernambuco. Revista Árvore, v. 38, n. 6, p. 1055-1064, 2014.

Ferreira, R. L. C. et al. Gestión forestal sostenible em la région semiárida de Brasil: actualización y perspectivas. In: Machuca, M. A. H. \& Ferreira, R. L. C. (Ed.). Gestión forestal sostenible: perspectivas y nuevos paradigmas frente al cambio global. Córdoba: Laboratorio de Gestión del Paisaje Forestal y Defensa contra Incendios, Universidad de Córdoba, 2010. p. 123-165.

Francelino, M. R. et al. Contribuição da caatinga na sustentabilidade de projetos de assentamentos no Sertão Norte-Rio-Grandense. Revista Árvore, v. 27, n. 1, p. 79-86, 2003.

Gomes, M. J. S. et al. Geração de renda e o manejo florestal em assentamentos de Pernambuco. Revista de Política Agrícola, v. 22, n. 4, p. 56-66, 2013.

Karvonen, J. et al. Indicators and tools for assessing sustainability impacts of the forest bioeconomy. Forest Ecosystems, v. 4, n. 2, 2017. DOI: 10.1186/s40663-017-0089-8.

Marques, M. W. C. F. et al. Composição de renda e a contribuição do manejo florestal em dois projetos de assentamento no Sertão de Pernambuco. REN: Revista Econômica do Nordeste, v. 42, n. 2, p. 247-258, 2011. Disponível em: http://www.bnb.gov.br/projwebren/ exec/artigoRenPDF.aspx?cd_artigo_ren=1240. Acesso: 12 jan. 2013.

Messier, C. \& Puettmann, K. J. Forest as complex adaptive systems: implications for forest management and modelling. Italian Journal of Forest and Moutains Environments, v. 66, n. 3, p. 249-258, 2011. DOI: 10.4129/ifm.2011.3.1.

Meunier, I. M. J. Análises de sustentabilidade de Planos de Manejo Florestal em Pernambuco. 2014. 135 f. Tese (Doutorado em Ciências Florestais) - Universidade Federal Rural de Pernambuco, Recife.
Meunier, I. M. J. et al. Curva ABC de estoque de densidade e volume aplicada ao manejo florestal de caatinga. Scientia Forestalis, v. 43, n. 106, p. 477-484, 2015.

Oliveira, A. D. et al. Avaliação econômica da regeneração da vegetação de cerrado, sob diferentes regimes de manejo. Revista Árvore, v. 26, n. 6, p. 715-726, 2002.

Pernambuco. Agência Estadual do Meio Ambiente. Instrução Normativa CPRH $\mathbf{n}^{0}$ 007/2006. Disciplina os procedimentos da CPRH referentes à aprovação da localização da Reserva Legal em propriedades e posses rurais; à autorização para supressão de vegetação e intervenção em Áreas de Preservação Permanente e à autorização para o desenvolvimento das atividades florestais no Estado de Pernambuco. Disponível em: <http://www.cprh.pe.gov. br/ARQUIVOS_ANEXO/IN\%20007\%202006;140606;20100420. pdf $>$. Acesso em: 12 jan. 2017.

Pukkala, T. Which type of forest management provides most ecosystem services? Forest Ecosystems, v. 3, n. 9, 2016. DOI: 10.1186/s40663-016-0068-5.

Riegelhaulpt, E. et. al. O manejo florestal na caatinga: resultados da experimentação. In: Gariglio, M. A. et al. Uso sustentável e conservação dos recursos florestais da Caatinga. Brasília, DF: Ministério do Meio Ambiente, Serviço Florestal Brasileiro, 2010. p. $256-275$.

Schneider, P. R. \& Finger, C. A. G. Manejo sustentado de florestas inequiâneas heterogêneas. Santa Maria, RS: Universidade Federal de Santa Maria, 2000.

Smith, R. L. \& Smith, T. M. Ecología. Madri: Pearson Educación, 2001.

Timofeicyzyk Junior, R. T. et al. Custo de oportunidade da terra no manejo de baixo impacto em florestas tropicais: um estudo de caso. Floresta, v. 37, n. 3, p. 327-338, 2007.

Timofeicyzyk Junior, R. T. et al. Rentabilidade econômica do manejo de baixo impacto em florestas tropicais: um estudo de caso. Floresta, v. 38, n. 4, p. 711-725, 2008.

Zakia, M. J. B. et al. Equações de peso e volume para oitos espécies lenhosas nativas do semi-árido, RN. Brasília, DF: IBAMA, 1990. (Circular técnica PNUD/FAO/BRA/87/007).

Zarin, D. J. Florestas produtivas neotropicais: conceitos e realidades. In: Zarin, D. J. et al. (Org.). As florestas produtivas nos neotrópicos: conservação por meio do manejo sustentável? São Paulo: Petrópolis, 2005. p. 19-32. 